\author{
Emine O. Tulunay \\ Faik C. Tulunay \\ Sinem E. Gulmez \\ Hakan Ergun \\ Alp Demireller
}

\section{The analgesic efficacy of dipyrone during removal of nasal packings after septal surgery}

Received: 30 March 2004

Accepted in revised form: 10 September 2004

This study has been presented at the 6th Congress of the European Association for Clinical Pharmacology and Therapeutics, 24-28 June 2003, Istanbul

\section{E.O. Tulunay • A. Demireller}

Department of Otorhinolaryngology Head and Neck Surgery,

Medical School of Ankara University, Ankara, Turkey

F.C. Tulunay $(\bowtie) \cdot$ S.E. Gulmez $\bullet$ H. Ergun Department of Pharmacology and Clinical Pharmacology,

Medical School of Ankara University,

Sihhiye, Ankara 06100, Turkey

e-mail: tulunay@dialup.ankara.edu.tr

Tel.: +90-312-3116495

Fax: +90-312-3116495

\begin{abstract}
Septoplasty is one of the most common nasal operations performed in otorhinolaryngological practice. At the end of the operation, most surgeons place nasal packings and remove them after 48-72 hours. The removal of the packings may be very painful. The objective of this study was to evaluate the analgesic effectiveness of dipyrone, when given before the procedure in a placebo-controlled, randomised design. Thirty-eight patients undergoing septal surgery at the Department of Otorhinolaryngology, Ankara University were included in this study. Twelve patients did not get any analgesic treatment during removal (control group). Ten patients received $2 \mathrm{ml}$ of intramuscular (i.m.) physiological saline solution (placebo group) and sixteen patients were pretreated with $1 \mathrm{~g}$ of i.m. dipyrone $45 \mathrm{~min}$ prior to the removal of the nasal packings (treatment group). Pain intensity
\end{abstract}

was measured prior to the procedure, just after the packings were removed $(0 \mathrm{~min})$ and then at 5,10 , $15,20,30,60$ and $120 \mathrm{~min}$ after the removal by using visual analogue scales, verbal scales and the percentage score. The groups were compared by using Kruskal-Wallis and Mann-Whitney tests. No significant difference in baseline pain scores was found before the procedure started. At 0, 5 and $10 \mathrm{~min}$ the dipyrone group showed significant lower pain intensity when compared to the control and placebo groups. Dipyrone was found to be effective in lowering initial pain intensity and in reducing it during the first 10 min after removal. We conclude that dipyrone is an effective agent when given before the procedure of removal of nasal packing-induced postprocedural pain.

Key words Septoplasty $\cdot$ Nasal packings $\cdot$ Dipyrone $\cdot$ Pre-emptive analgesia

\section{Introduction}

Packing in nasal surgery is primarily used to control bleeding and to obtain internal stabilisation of the operation site.
Mainly after septal operations, nasal packing helps to minimise the dead space and thus prevent the formation of septal haematomas and abscess, as well as synechias in the long term. There are not any widely accepted rules regarding which packing material should be used or how long 
they should be left in the nasal cavity. Every surgeon has a preference and some do not use packing at all. One of the main concerns about using packing is that the removal is usually very painful and can be very bothersome. This procedure may even result in syncope by the activation of the vasovagal reflex system. Unfortunately, this very intense pain is usually inadequately treated.

Administration of analgesic agents before the painful procedure is used widely in many different surgical fields. In this method, analgesics are administered prior to the procedure, which is the noxious stimulus, in order to prevent central sensitisation and limit the subsequent pain experience [1]. Thus, preventing the initial cascade could lead to long term benefits by eliminating the hypersensitivity produced by the noxious stimuli [1].

Although it has been studied in many fields, whether it has any beneficial effects after nasal surgeries has not been evaluated thoroughly. In this study we aimed to investigate the effects of dipyrone administered prior to the removal of nasal packing on pain caused by the procedure, in a randomised, placebo controlled study design.

\section{Material and methods}

Thirty-eight patients undergoing septal surgery (septal and/or turbinate surgery) at the Department of Otorhinolaryngology, Ankara University were included in the study. The study was performed in accordance with the Declaration of Helsinki and with the local laws and regulations relevant to the use of new and approved therapeutic agents in patients. The protocol was approved by the local ethics committee of the centre, and conducted according to the International Conference on Harmonization-Good Clinical Practice (ICH-GCP) standard. All patients provided written informed consent before their enrolment in the study.

Gauze packings impregnated with soft paraffin were used and were kept in the nasal cavity for 48 hours. This routine intervention in our department is not strict and patients usually do not receive any medication prior to the removal of the packings, and are treated with pain killers after the onset of pain. The 38 patients were randomised into 3 groups. The first group comprised of 12 patients that did not receive any analgesic treatment or intervention during removal (control group). In the second group, 10 patients received $2 \mathrm{ml}$ of intramuscular (i.m.) physiological saline solution $45 \mathrm{~min}$ prior to removal of the nasal packings (placebo group). The third group included 16 patients pretreated with $1 \mathrm{~g}(2 \mathrm{ml})$ of i.m. dipy- rone 45 min prior to the removal of the nasal packings (treatment group). The patients were asked to express their pain intensity by using visual analogue scales (VAS), verbal scales (VS) and the percentage score (PS). The scales were filled out prior to the procedure, just after the packings were removed $(0 \mathrm{~min})$ and then at 5 , $10,15,20,30,60$ and 120 min after the removal. The VAS was a $100 \mathrm{~mm}$ long line with " 0 " (no pain), and "10" (the worst pain that can be imagined) marked on both ends. The verbal rating system was as follows: "0" (no pain), "1" (mild pain), "2" (moderate pain), " 3 " (severe pain) and " 4 " (very severe pain). The patients were allowed to rest at the hospital for 2 hours and were discharged after 2 hours if they had no bleeding or any other complications. The Kruskal-Wallis non-parametric analysis of variance was used to test for significant differences between the three groups. The Mann-Whitney test was used to disclose a statistically significant difference between groups at $p<0.05$.

\section{Results}

There was no difference between the study groups according to age, gender and medical history. Demographic data of the three groups are tabulated in Table 1.

No significant difference in baseline pain scores was found before the procedure started. At 0,5 and $10 \mathrm{~min}$, the dipyrone group showed significant pain relief when compared to the control and placebo groups (Fig. 1).

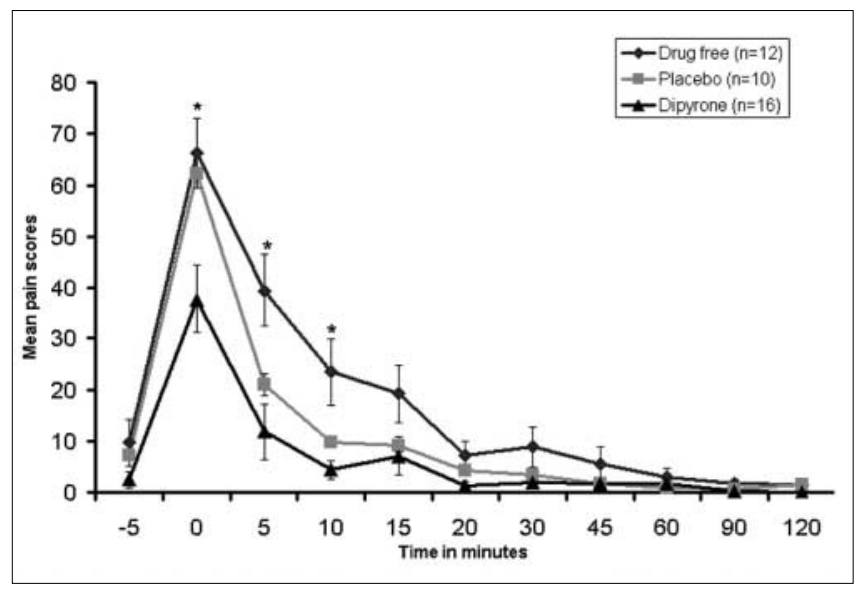

Fig. 1 Mean pain scores measured using $100 \mathrm{~mm}$ Visual Analogue Scale. Time point " 5 " and "0" represent the pain scores before and right after the removal of nasal packing, respectively

Table 1 Demographic data of the enrolled patients

\begin{tabular}{llll}
\hline Group & $\mathrm{n}$ & Gender & Age $( \pm$ SD) \\
\hline Control & 12 & $6 \mathrm{M}, 6 \mathrm{~F}$ & $34( \pm 11)$ \\
Placebo & 10 & $6 \mathrm{M}, 4 \mathrm{~F}$ & $35( \pm 11)$ \\
Treatment (dipyrone) & 16 & $7 \mathrm{M}, 9 \mathrm{~F}$ & $32( \pm 10)$ \\
\hline
\end{tabular}


There was a good correlation between the methods used to measure the pain intensity. The correlation coefficients were as follows: VAS-VS: 0.77; VAS-PS: 0.93; PS-VS: 0.85 .

None of the patients enrolled in the study reported, nor did physicians observe, any adverse effect during the 2hour observation period.

\section{Discussion}

The concept of administering analgesics before the painful procedure to reduce the magnitude and duration of postoperative pain was paved in 1983 by Wolf, who showed evidence for a central component of postinjury pain hypersensitivity in experimental studies [2]. After this, an overwhelming amount of experimental data demonstrated that various antinociceptive techniques applied before injuries were more effective in reducing the postinjury central sensitisation phenomena as compared with administration after injury [2].

Preemptive analgesia is based on the observations that if the trigger signals were prevented from gaining access to the central nervous system (CNS), pain would be diminished, and that treatment aimed at reducing any enhanced excitability within the CNS initiated by a trigger would reduce hyperalgesia and allodynia [3]. Two methods are available for preventing central sensitisation: conduction blockade with local anaesthetics so that the signal from the injured region does not reach the spinal cord; and suppression of the excitability of the nervous system before it receives the nociceptive input [3].

In spite of all these innovations in relieving pain, physicians still have a poor track record for relieving pain. A large number of studies document this failure, particularly in patients undergoing surgery and patients experiencing cancer-related pain [4]. Although it is a well known fact that patients who undergo septal surgery may experience very severe pain during the removal of nasal packings, only a few studies have been conducted to obtain a standard procedure [5].

Little has been published on nasal packings and even less about how to relieve the pain caused by their removal. Von Schoenberg et al. reported that the removal of the packings was the most painful event postoperatively [6].

Lavy et al. examined the efficacy of removing Merocel packings after rehydrating them with a local anaesthetic solution, lignocaine, in a study of 34 patients. In their study, in which each patient served as his own control, they could not find any statistically significant difference between rehydration with lignocaine solution or normal saline [7]. Kuo et al., on the other hand, used lignocaine mixed vaseline gauze packings to assess the effects of local anaesthetics on pain while the packings were in the nasal cavity and during removal. Although the lignocaine group required significantly less oral analgesics after the operation, the results were only statistically significant for the first 3 hours and did not show any significant effect on removal [8].

Laing et al. randomised 150 patients into 3 groups. Group 1 received no treatment, group 2 received i.m. papaveretum $30 \mathrm{~min}$ prior to removal and group 3 received Etonox just before removal. They reported significant success with Etonox; also, the pain scores of the papaveretum group were significantly better than that of the no treatment group [9]. The disadvantages of this method are that nursing staff need to be trained how to administer Etonox, which causes time consumption and higher cost than simply administering an analgesic.

Some authors have reported significantly reduced pain without the use of packings. Von Schoenberg et al. stated that they found the use of nasal packings hard to justify, as they found significantly lower rates of pain in the patients when they used septal suturing instead of packing. Besides, most of the complications in their study were related to the packings [6]. Nunez et al. reported similar results and encouraged the use of septal quilt sutures instead of nasal packing [10].

Despite the controversies on using packings, many surgeons still believe a nasal packing to be necessary, which is also the routine procedure in our department. Apart from stabilising the surgical field and preventing haemorrhage, Thomas et al. draws attention to the importance of preventing the drainage of the blood into the oropharynx during the first couple of hours after the operation when the patient is not fully conscious and faces the risk of aspiration [11].

Nonsteroidal anti-inflammatory drugs (NSAIDs) are gaining popularity in the management of pain associated with ambulatory surgery. In an effort to minimise the bleeding complications, gastrointestinal and renal damage associated with classical NSAIDs, dipyrone was chosen to achieve pain control during the removal of the packings. Dipyrone was administered 45 min before the procedure in order to achieve an appropriate therapeutic plasma concentration according to its pharmacokinetic profile [12].

In this study, we used an analgesic already available throughout all of the wards in the hospital. The aim was to suppress the excitability of the nervous system before it received any stimulus. Therefore, dipyrone was administered 45 min before removal of the packings to achieve an appropriate blood concentration. We found significant lower pain intensities during removal and in the first 10 min. This is the period in which the patients experience most of the pain, as seen from the data of the group that 
received no treatment (Fig. 1). Saving the patient from such a stressful experience by simply administering dipyrone would make future surgery much more acceptable if needed. We also know that the most unpleasant aspect of nasal surgery is the postoperative removal of the nasal packings. There are many patients who decide not to have septal surgery on the basis of this fact.

As a result, the aim of every physician should be minimising the discomfort of the patient as much as possible.
Administering an analgesic prior to removal is a very effective and also cost-effective method. To minimise the bleeding complications, gastrointestinal and renal damage associated with classical NSAIDs, dipyrone may be a good alternative. The patients in the dipyrone group experienced significantly lower pain than the control and the placebo groups. This seems to be a promising pharmacological intervention and needs to be evaluated in detail and in comparison with other effective NSAIDs in further studies.

\section{References}

1. Gottschalk A, Smith DS (2001) New concepts in acute pain therapy: preemptive analgesia. Am Fam Physician 63:1979-1984

2. Moiniche S, Kehlet H, Dahl JB (2002) A qualitative and quantitative systemic review of preemptive analgesia for postoperative pain relief: the role of timing of analgesia. Anesthesiology 96(6):725-741

3. Suzuki H (1995) Recent topics in the management of pain: development of the concept of preemptive analgesia. Cell Transplant 4(1):3-6

4. Siwek J (2001) Preemptive analgesia: decreasing pain before it starts. Am Fam Physician 63(10):1924
5. Weber R, Keerl R, Hochapfel F, Draf W, Toffel PH (2001) Packing in endonasal surgery. Am J Otolaryngol 22(5):306-320

6. Von Schoenberg M, Robinson P, Ryan R (1993) Nasal packing after routine nasal surgery - is it justified? J Laryngol Otol 107:902-905

7. Lavy JA, Small GVB, Radcliffe GJ (1996) A prospective randomized controlled study of $4 \%$ lignocaine solution in merocel nasal pack removal. Rhinology 34:219-221

8. Kuo MJ, Zeitoun H, Macnamara M, Wagstaff K, Carlin WV, Turner N (1995) The use of topical 5\% lignocaine ointment for the relief of pain associated with postoperative nasal packing. Clin Otolaryngol 20:357-359
9. Laing MR, Clark LJ (1990) Analgesia and removal of nasal packing. Clin Otolaryngol 15:339-342

10. Nunez DA, Martin FW (1991) An evaluation of post-operative packing in nasal septal surgery. Clin Otolaryngol 16:549-550

11. Thomas DM, Samuel D, Tierney PA, Patel KS (1996) Audit of pain after nasal surgery. Ann R Coll Surg Engl 78:380-382

12. Levy M, Zylber-Katz E, Rosenkranz B (1995) Clinical pharmacokinetics of dipyrone and its metabolites. Clin Pharmacokinet 28(3):216-234 